\title{
Proposal and Analysis of a New Spectrum Sensing Algorithm for Cognitive Radio Driven Hospitals
}

\author{
Amir Eslami ${ }^{*}$, Saeid Karamzadeh ${ }^{1}$
}

Accepted $3^{\text {rd }}$ September 2016

\begin{abstract}
Wireless technology is the key technology to eliminate the dense wire ropes from hospitals and far access to medical devices. In order to overcome the problem of bandwidth scarcity, cognitive radio driven hospitals are introduced and devices are divided in two categories. The first category is primary devices and the second one is secondary devices. Primary devices has very high priority and their communication is vital for the hospital and patients, so that no interference should be made with such devices. Secondary devices are the ones which has lower priority and they can wait until the primary devices do their communication and then, they begin to use the allocated spectrum. One of the key functions to assure that there will be no interference is a reliable spectrum sensing method. This method should be a simple one to be able to implement it in the secondary devices. Among all the sensing methods, energy detection (ED) based spectrum sensing is very popular. In order to improve the performance of ED, double threshold ED (DTED) method is introduced in literature. In this paper, a new algorithm is intruded for DTED considering previous sensing period results in detection procedure by using a memory stick. Memoryful DTED (MDTED) improves the performance of DTED considerably by only the cost of delay in secondary devices communication which has less value than the improvement of the detection method performance.
\end{abstract}

Keywords: Wireless technology hospitals, Cognitive radio driven hospitals, Spectrum sensing, Energy detection, Double threshold energy detection, Memory.

\section{Introduction}

It has been a long while that biomedical and e-health experts are trying to use wireless technologies in their field. The main purpose of this matter, instead of eliminating the dense wire ropes from the hospital environment, is to provide access from distance to the devices in the environment. To meet this requirement, the number of wireless technology based hospitals grow so fast that after a while the problems began to appear. One of the most important problems was the scarcity of bandwidth. The bandwidth of the medical wireless communication is limited because of static frequency allocation which is done by governmental and non-governmental commissions. In United States, the responsibility of spectrum allocation is with Federal Communications Commission (FCC). In order to mitigate the scarcity of the spectrum, cognitive radio driven hospitals (CogMed) are introduced by researchers. In this kind of hospitals, devices are categorized as primary and secondary devices. Primary devices are the ones that are vial and have higher priority to have communication. Secondary devices are the ones which have low priority and thy can wait until the frequency band get vacant. So that, primary devices should not be interference by other devices as their data are so valuable. In cognitive radio technology, the secondary users sense the bandwidth and in the case of vacancy, the begin communication, otherwise, they wait for predetermined moment and sense the frequency band again. There are many sensing algorithms including ED, matched filtering, wavelet based detection, cyclostationary detection and covariance based detection methods with their different required parameters, advantages and disadvantages. For instance,

\footnotetext{
${ }^{1}$ Electric and Electronics Engineering Department, Istanbul Aydin University, Istanbul/Turkey

* Corresponding Author: Email: eslami15@itu.edu.tr

Note: This paper has been presented at the $3^{\text {rd }}$ International Conference on Advanced Technology \& Sciences (ICAT'16) held in Konya (Turkey), September 01-03, 2016.
}

cyclostationary sensing methods needs the data about the cyclic frequency of the primary user and in matched filter sensing method, the waveform information is needed. As mentioned before, each sensing method has its own advantages and disadvantages that makes them unique in their kind, but in practice, the most important factor is the simplicity of the used method. Among all the methods mentioned, ED method is the simplest one to use in devices. ED method is based on comparison of the energy of collected samples with a threshold calculated before. In order to improve the performance of ED sensing method, cooperative sensing method and DTED are introduced in literature. Cooperative ED spectrum sensing is not practical solution for CogMed as there are many devices and in the case that each device use multiple sensing nodes, too complicated and busy environment will be the result of this process. The second solution is using double threshold instead of single one. In double threshold the detection is made comparison of the energy of the signal with two pre-calculated thresholds. This process has improved the performance of ED method considerably, but for CogMed usage, as the inter symbol interference (ISI) with primary users could cost somebodies life, the performance should be much higher with little additional computational complexity. In this paper we recommend to use memory for the secondary user devices, so that, in the detection procedure, they could consider the result of the previous sensing period. By MDTED, in the case of any error in the sensing period, the previously gathered information will help the algorithm to check the status of the channel previously. The rest of the paper is organized as follows. In section II, The system model, ED method and some background information are provided. In section III, DTED and MDTED methods are introduced. In Section IV simulation results and analysis is done, followed by concluding remarks in section V.

\section{Energy Detection, System Model and Background}


The spectrum procedure in cognitive radio secondary medical devices is a binary hypothesis. Hypothesis $\mathrm{H}_{1}$ is the condition which the primary user is having a data transfer and in the case of beginning communication, interference will occur. Hypothesis $\mathrm{H}_{0}$ is when the primary user doesn't use the spectrum and secondary -user can use the spectrum. These two hypothesis can be shown as follow:

$$
y(t)= \begin{cases}\eta(t) & : H_{0} \\ h x(t)+\eta(t) & : H_{1}\end{cases}
$$

where $y(t)$ is the received signal by secondary user, $x(t)$ is the signal used for primary user, $h$ is the communication channel gain and $\eta(t)$ is additive white Gaussian noise (AWGN) which is assumed to be stationary process that satisfy $E(\eta(t))=0$ and $E(\eta(t)$ $\eta(t+\tau))=0$ for any $\tau \neq 0$ and variance equal to $\sigma_{\eta}^{2}$. Nakagami-m fading distribution is the most used distribution in real life communication wireless channels. This model can be represented as below:

$\mathrm{P}_{\rho}=\frac{2 m^{m} x^{2 m-1}}{\Gamma(\mathrm{m}) \Omega^{m}} \exp \left(-\frac{m x^{2}}{\Omega}\right), \mathrm{m} \geq \frac{1}{2}, \mathrm{x} \geq 0$

$\Gamma(\mathrm{m})=\int_{0}^{\infty} y^{m-1} e^{-y} d y$

$$
\text { , } m>0
$$

that $\mathrm{m}$ varies based on the channel characteristics. A Rayleigh fading channel can be modelled when $\mathrm{m}$ is equal to one and the a Gaussian channel can be modelled when $\mathrm{m}$ goes to infinity. $\Gamma($.) is a gamma function.

Energy detection based spectrum sensing detector is consist of a pre-filtering system, square law device and a finite time integrator. The output of the integrator is normalized and can be shown as follow:

$\mathrm{E}(\mathrm{t})=\frac{1}{N} \sum_{n=0}^{N-1}|x(n)|^{2}$

where $\mathrm{N}$ is the number of samples collected by the secondary user receiver. As the received signals are unknown, the gathered samples can be treated as random processes. So, the transmitted signal samples follows and independent and identically distributed (i.i.d.) random processes with mean equal to zero and variance equal to $\sigma_{x}^{2}$. The received signal to noise ratio (SNR) at the detector which we show it by $\beta$ symbol from now on is equal to the amount mentioned below.

$\beta=\frac{|h|^{2} \sigma_{x}^{2}}{\sigma_{\eta}^{2}}$

Assuming the number of collected signals large enough, using central limit theorem, the probability density function (PDF) of $\mathrm{E}(\mathrm{t})$ under hypothesis $\mathrm{H}_{0}$ becomes a normal distribution with mean equal to $\mathrm{N} \sigma_{\eta}^{2}$ and variance equal to $\mathrm{N} \sigma_{\eta}^{4}$. PDF of $\mathrm{E}(\mathrm{t})$ under hypothesis $\mathrm{H}_{1}$ becomes a normal distribution with mean equal to $\mathrm{N}(1+\beta) \sigma_{\eta}^{2}$ and variance equal to $(1+2 \beta) \mathrm{N} \sigma_{\eta}^{4}$. The probability that the detector detects the presence of signal under hypothesis $\mathrm{H}_{0}$ is called probability of false alarm $\left(\mathrm{P}_{\mathrm{fa}}\right)$ and the probability that the detector detects the presence of signal under hypothesis $\mathrm{H}_{1}$ is called probability of detection $\left(\mathrm{P}_{\mathrm{d}}\right)$, can be shown as follow:

$\beta=\frac{|h|^{2} \sigma_{x}^{2}}{\sigma_{\eta}^{2}}$

$\mathrm{P}_{\mathrm{fa}}=\operatorname{prob}\left(\mathrm{E}(\mathrm{t})>\lambda \mid \mathrm{H}_{0}\right)=\Gamma\left(\mathrm{u}, \frac{\lambda}{2}\right) / \Gamma(\mathrm{u})=\mathrm{Q}\left(\frac{\lambda-\sigma_{\eta}^{2}}{\sqrt{2 \sigma_{\eta}^{4} / N}}\right)$

$\mathrm{P}_{\mathrm{d}}=\operatorname{prob}\left(\mathrm{E}(\mathrm{t})>\lambda \mid \mathrm{H}_{1}\right)=\mathrm{Q}_{\mathrm{u}}(\sqrt{2 \alpha}, \sqrt{\lambda})=\mathrm{Q}\left(\frac{\lambda-\left(|h|^{2} \sigma_{x}^{2}+\sigma_{\eta}^{2}\right)}{\sqrt{2\left(|h|^{2} \sigma_{x}^{2}+\sigma_{\eta}^{2}\right) / \mathrm{N}}}\right)$

In IEEE802.22, the $\mathrm{P}_{\mathrm{fa}}$ is given but for different values, threshold based on $\mathrm{P}_{\mathrm{fa}}$ which we are going to simply call it threshold can be calculated as follow:

$\lambda_{\mathrm{fa}}=\boldsymbol{\sigma}_{\eta}{ }^{2}\left(1+\frac{\sqrt{2} Q^{-1}\left(p_{f a}\right)}{\sqrt{N}}\right)$

and the threshold based on $\mathrm{P}_{\mathrm{d}}$ at a given SNR can be shown as follow:

$\lambda_{\mathrm{d}}=\boldsymbol{\sigma}_{\eta}{ }^{2}(1+\alpha)\left(1+\frac{\sqrt{2} Q^{-1}\left(p_{d}\right)}{\sqrt{N}}\right)$

Based on ED algorithm, the normalized energy of the gathered signal samples is compared with the pre-calculated threshold and in the case that the normalized energy is equal or bigger than the threshold, cognitive radio based secondary device detects the presence of the primary user and doesn't start its communication. In the case that the normalized energy is less than the threshold, it begins its communication.

\section{Double Threshold Energy Detection and Memoryful Double Threshold Energy Detection}

DTED method is introduced in literature to improve the detecting performance of ED and make this method more reliable. By a little error, the sample energy can pass the threshold or can be less than the threshold defined. The main purpose in this sensing algorithm is defining a restricted area near threshold that contains these faulty samples and eliminate them from the detection process as they are more likely to cause an error. Restricted area constant (RAC) is shown as $\Delta$ and defined as below:

$\lambda_{1}=(1-\Delta) \lambda$

As lower boundary

$\lambda_{2}=(1+\Delta) \lambda$

As higher boundary

In DTED algorithm, the samples are lower than $\lambda_{1}$ and higher than $\lambda_{2}$ are used for detection process. If the normalized energy of the received signals is lower than $\lambda_{1}$ the secondary medical device concludes that the spectrum is vacant and in the case that the received signal is higher than the value of $\lambda_{2}$, the decision is accuracy of it. In DTED method, the probability of the energy be between boundaries in condition of hypothesis $\mathrm{H}_{0}$ is shown as $\mathrm{P}_{0}$ and in the case that the probability of the energy be between boundaries in condition of hypothesis $\mathrm{H}_{1}$ is shown as $\mathrm{P}_{1} . \mathrm{P}_{\mathrm{fa}}, \mathrm{P}_{\mathrm{d}}$, $\mathrm{P}_{1}$ and $\mathrm{P}_{0}$ probabilities can be shown as follow:

$\mathrm{P}_{\mathrm{fa}}=\mathrm{p}\left(\mathrm{E}\left(\mathrm{N}_{\mathrm{s}}\right)>\lambda_{2} \mid \mathrm{H}_{0}\right)=\Gamma\left(\mathrm{u}, \frac{\lambda 2}{2}\right) / \Gamma(\mathrm{u})$

$\mathrm{P}_{\mathrm{d}}=\mathrm{p}\left(\mathrm{E}\left(\mathrm{N}_{\mathrm{s}}\right)>\lambda_{2} \mid \mathrm{H}_{1}\right)=\mathrm{Q}_{\mathrm{u}}\left(\sqrt{2 \beta}, \sqrt{\lambda_{2}}\right)$

$\mathrm{P}_{0}=\mathrm{p}\left(\lambda_{1}<\mathrm{E}\left(\mathrm{N}_{\mathrm{s}}\right)<\lambda_{2} \mid \mathrm{H}_{0}\right)=\left(\Gamma\left(\mathrm{u}, \frac{\lambda 1}{2}\right) / \Gamma(\mathrm{u})\right)-\left(\Gamma\left(\mathrm{u}, \frac{\lambda 2}{2}\right) / \Gamma(\mathrm{u})\right)$

$\mathrm{P}_{1}=\mathrm{p}\left(\lambda_{1}<\mathrm{E}\left(\mathrm{N}_{\mathrm{s}}\right)<\lambda_{2} \mid \mathrm{H}_{1}\right)=\mathrm{Q}_{\mathrm{u}}\left(\sqrt{2 \beta}, \sqrt{\lambda_{1}}\right)-\mathrm{Q}_{\mathrm{u}}\left(\sqrt{2 \beta}, \sqrt{\lambda_{2}}\right)$

As mentioned earlier, reliability is the most important factor in CogMed and having multiple detectors for having cooperative detection is not possible. MDTED is a novel approach to DTED which improves the detection performance of it. This algorithm is based on saving the energy of last $\mathrm{T}$ previously sensed signal samples and detection is made considering these energies. Collection of $\mathrm{T}$ consecutive energy samples can be shown as follow:

$M_{i}\left(y_{i}\right)=\left\{E\left(y_{i-T}\right), E\left(y_{i-T+1}\right), E\left(y_{i-T+2}\right), \ldots, E\left(y_{i}\right)\right\}$

In the case that the maximum of these decision energies fall below the threshold $\lambda_{1}$ the detection process decides the hypothesis $\mathrm{H}_{0}$ and in the case that the maximum is higher than $\lambda_{2}$ the detection process decides the hypothesis $\mathrm{H}_{1}$. If the maximum is between $\lambda_{1}$ and $\lambda_{2}$ in any way, the next maximum value is going to be used until it doesn't be in the restricted gap. Because of environment in real life doesn't change rapidly and in high order by time, this method will help our detection system to not only have the results of the time, it will use previously gathered information also that will help decision process in big range. Considering the improvement of detection process performance and reliability, T-1 sensing period delays can be bearable. Also by choosing $\mathrm{T}$ small enough, it is possible to make the delay minimum. The algorithm of MDTED is as follow:

Algorithm 1. MDTED spectrum sensing method

Input $\quad: \mathrm{T}, \lambda_{1}, \lambda_{2}, \sigma_{\eta}$

Output $: \mathrm{Y}_{\mathrm{i}}$

1: $\quad$ for each sensing period do

2: $\quad$ for $\zeta=1: \mathrm{N}$ samples do

3: $\quad$ if $\lambda_{1<\text { sample energy }<\lambda_{2}}$ 
4: $\quad$ do nothing

5: $\quad$ else

6: $\quad \mathrm{e}_{\zeta}(\mathrm{t}) \leftarrow$ Energy of sample $\zeta, \zeta \in[1,2, \ldots, \mathrm{N}]$

7: $\quad$ end for

8: $\quad \mathrm{e}(\mathrm{t})=$ normalized energy of the selected total $\mathrm{e}_{\zeta}(\mathrm{t})$ samples

9: $\quad \mathrm{M}_{\mathrm{i}}\left(\mathrm{y}_{\mathrm{i}}\right) \leftarrow$ Energy of previous $\mathrm{T}$ received signals and $\mathrm{e}(\mathrm{t})$

10: $\quad \mathrm{M}(\mathrm{t})=\operatorname{MAX}\left\{\mathrm{M}_{\mathrm{i}}\left(\mathrm{y}_{\mathrm{i}}\right)\right\}$

11: $\quad$ if $\lambda_{1<M} \mathrm{M}(\mathrm{t})<\lambda_{2}$

12: $\quad \mathrm{Q}_{\mathrm{i}}\left(\mathrm{y}_{\mathrm{i}}\right)=\mathrm{M}_{\mathrm{i}}\left(\mathrm{y}_{\mathrm{i}}\right)-\mathrm{M}(\mathrm{t})$

13: $\quad \mathrm{M}(\mathrm{t})=\operatorname{MAX}\left\{\mathrm{Q}_{\mathrm{i}}\left(\mathrm{y}_{\mathrm{i}}\right)\right\}$

14: $\quad$ end if

15: $\quad$ if $\mathrm{M}(\mathrm{t})<\lambda_{1}$ then

16: $\quad \mathrm{Y}_{\mathrm{i}} \leftarrow \mathrm{H}_{0}$

17: $\quad$ else

18: $\quad \mathrm{Y}_{\mathrm{i}} \leftarrow \mathrm{H}_{1}$

19: $\quad$ return $Y_{i}$

20: $\quad$ end for

$\mathrm{P}_{\mathrm{d}}, \mathrm{P}_{\mathrm{fa}}, \mathrm{P}_{0}$ and $\mathrm{P}_{1}$ can be calculated as follow:

$\mathrm{P}_{\mathrm{fa}}=\mathrm{p}\left(\mathrm{M}(\mathrm{t})>\lambda_{2} \mid \mathrm{H}_{0}\right)=\mathrm{p}\left(\mathrm{E}\left(\mathrm{y}_{\mathrm{i}}\right)>\lambda_{2}, \mathrm{E}\left(\mathrm{y}_{\mathrm{i}-1}\right), \ldots, \mathrm{E}\left(\mathrm{y}_{\mathrm{i}^{-} \mathrm{T}}\right)>\lambda_{2} \mid \mathrm{H}_{0}\right)=$

$\mathrm{p} \quad\left(\mathrm{E}\left(\mathrm{y}_{\mathrm{i}}\right)>\lambda_{2} \mid \mathrm{H}_{0}\right) \cdot \mathrm{p}\left(\mathrm{E}\left(\mathrm{y}_{\mathrm{i}-1}\right)>\quad \lambda_{2} \mid \mathrm{H}_{0}\right) \quad \ldots \quad \mathrm{p}\left(\mathrm{E}\left(\mathrm{y}_{\mathrm{i}}-\mathrm{c}\right)>\quad \lambda_{2} \mid \mathrm{H}_{0}\right)=$ $\mathrm{Q}\left(\frac{\lambda_{2}-\sigma_{\eta}^{2}}{\sqrt{2 \sigma_{\eta}^{4} / N}}\right)^{\mathrm{T}+1}$

$\mathrm{P}_{\mathrm{d}}=\mathrm{p}\left(\mathrm{M}(\mathrm{t})>\lambda_{2} \mid \mathrm{H}_{1}\right)=\mathrm{p}\left(\mathrm{E}\left(\mathrm{r}_{\mathrm{i}}\right)>\lambda_{2}\left|, \mathrm{E}\left(\mathrm{r}_{\mathrm{i}-1}\right), \ldots, \mathrm{E}\left(\mathrm{r}_{\mathrm{i}}-\mathrm{c}\right)>\lambda_{2}\right| \mathrm{H}_{1}\right)=$

$\mathrm{p} \quad\left(\mathrm{E}\left(\mathrm{r}_{\mathrm{j}}\right)>\lambda_{2} \mid \mathrm{H}_{1}\right) \cdot \mathrm{p}\left(\mathrm{E}\left(\mathrm{r}_{\mathrm{i}-1}\right)>\lambda_{2} \mid \mathrm{H}_{1}\right) \quad \ldots \quad \mathrm{p}\left(\mathrm{E}\left(\mathrm{r}_{\mathrm{i}}-\mathrm{c}\right)>\lambda_{2} \mid \mathrm{H}_{1}\right)=$ $\mathrm{Q}\left(\frac{\lambda_{2}-\left(|h|^{2} \sigma_{s}^{2}+\sigma_{\eta}^{2}\right)}{\sqrt{2\left(|h|^{2} \sigma_{s}^{2}+\sigma_{\eta}^{2}\right) / \mathrm{N}}}\right)^{\mathrm{T}+1}$

$\mathrm{P}_{0}=\mathrm{p}\left(\lambda_{1}<\mathrm{M}(\mathrm{t})<\lambda_{2} \mid \mathrm{H}_{0}\right)=\left(\mathrm{Q}\left(\frac{\lambda_{1}-\sigma_{\eta}^{2}}{\sqrt{2 \sigma_{\eta}^{4} / N}}\right)-\mathrm{Q}\left(\frac{\lambda_{2}-\sigma_{\eta}^{2}}{\sqrt{2 \sigma_{\eta}^{4} / N}}\right)\right)^{\mathrm{T}+1}$

$\mathrm{P}_{1}=\mathrm{p}\left(\lambda_{1}<\mathrm{M}(\mathrm{t})<\lambda_{2} \quad \mid \mathrm{H}_{1}\right)=\left(\mathrm{Q}\left(\frac{\lambda_{1}-\left(|h|^{2} \sigma_{s}^{2}+\sigma_{\eta}^{2}\right)}{\sqrt{2\left(|h|^{2} \sigma_{s}^{2}+\sigma_{\eta}^{2}\right) / \mathrm{N}}}\right)\right.$

$\left.\mathrm{Q}\left(\frac{\lambda_{2}-\left(|h|^{2} \sigma_{S}^{2}+\sigma_{\eta}^{2}\right)}{\sqrt{2\left(|h|^{2} \sigma_{S}^{2}+\sigma_{\eta}^{2}\right) / \mathrm{N}}}\right)\right)^{\mathrm{T}+1}$

MBED decision performance is analysed and compared with DTED method in section IV.

\section{Simulation Results and Analysis}

In this section the performance of MDTED is studied, analysed and compared with very well-known DTED. All simulations are done in MATLAB software using QPSK modulated random signals and i.i.d. noise samples with Gaussian random variables. It is assumed that the channel doesn't change while sampling in each sampling period. Based on IEEE 802.22, $\mathrm{P}_{\mathrm{fa}}$ should be less or equal to 0.1 and $\mathrm{P}_{\mathrm{d}}$ be more than 0.9. In the simulations $\mathrm{P}_{\mathrm{fa}}$ is chosen as 0.1 and $10^{5}$ test signals are used and averaged. DTED and MDTED is studied with RAC equal to 0.5 and in memory section, $\mathrm{T}$ is equal to 2 unless it is told to be different. T equal to 2 mean the detection method needs 2 previously sensed normalized energies that should be saved in memory and be used in the detection procedure.

Figure 1 shows the performance of ED, DTED and MDTED in Gaussian channel models. Gaussian channel is the simplest channel model among all communication channel models.

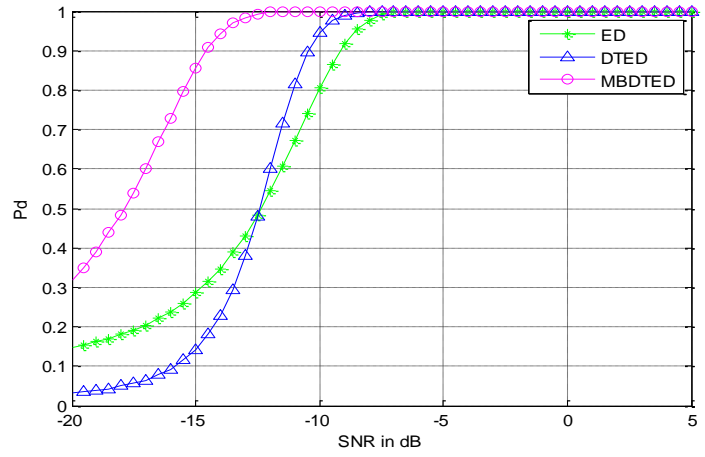

Figure 1. ED, DTED and MBDTED sensing methods performance over Gaussian channel

MBDTED with a memory equal to 2, has a much better performance compared to DTED and ED in all SNR ranges. With only saving 2 previously sensed signals energy in detection process, a high range of performance can be achieved. Unlike DTED, the performance of MBDTED doesn't diminish suddenly in low SNRs like DTED.

Figure 2 show the performance of ED, DTED and MBDTED in Rayleigh channel which is known as one of the worst communication channels. In this kind of channel, MBDTED again has a better performance compared to both DTED and ED also in all SNR ranges by only the delay of 2 sensing periods.

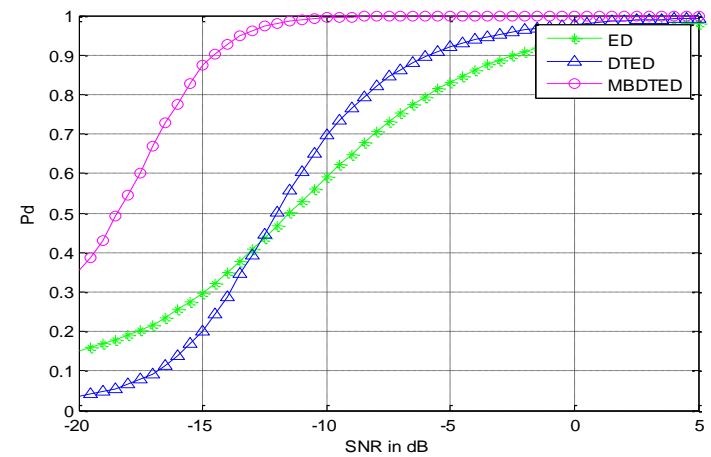

Figure 2. ED, DTED and MBDTED sensing methods performance over Rayleigh channel

Figure 3 shows the performance of MBDTED whith different memory values in Gaussian channels. In MBDTED without memory is the original DTED. By only using 1 previously sensed signal energy the detection performance gets much higher. This value is getting better when we choose the T value equal to 2 .

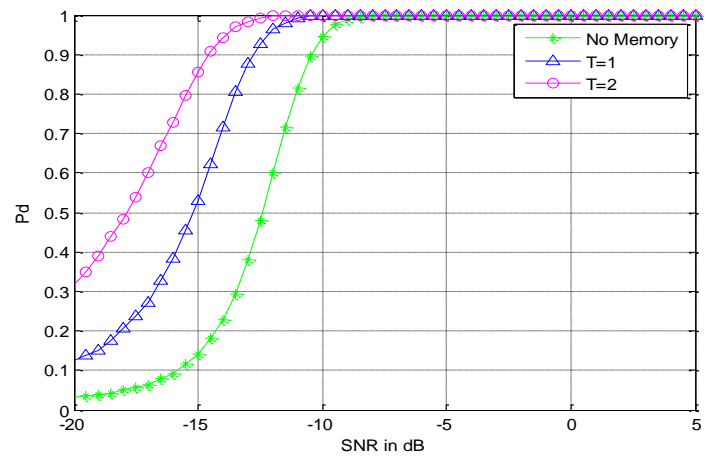

Figure 3. MBDTED sensing method performance without memory and memory of saving 1 and 2 previously sensed signals energy over Gaussian channel. 


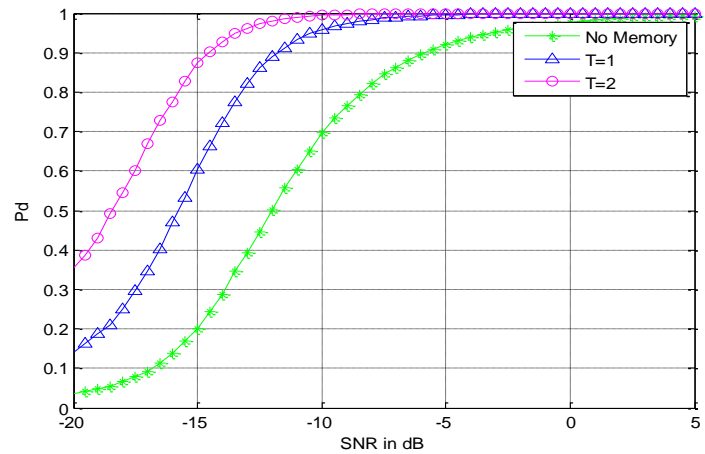

Figure 4. MBDTED sensing method performance without memory and memory of saving 1 and 2 previously sensed signals energy over Rayleigh channel.

In figure 4 , the performance of MBDTED is evaluated in different memory values in Rayleigh channel. This can be seen from the figure that in this kind of channel model, again the performance is getting better as the $\mathrm{T}$ value gets higher.

Choosing the value of memory, based on the communication channel estimated in the cognitive radio driven hospitals and acceptable delay in the sensing procedure, we can have the best performance needed in such areas.

\section{Conclusion}

Memory based double threshold energy detection or in other words, double threshold energy detection with memory sensing algorithm is introduced in this paper. It is shown that using memory and saving the previously received signals energy in the defined algorithm can affect the sensing performance and improve the detection performance of the energy detection based spectrum sensing in very high range. DTED was defined in literature to improve the performance and reliability of ED method. We compared the MBDTED with ED and DTED methods in Gaussian and Rayleigh channels by using only 2 previously saved signals and we saw the performance of this method is much higher than both ED and DTED in wide SNR range. Disadvantage of this method is the time delay after the channel is free but considering the improvement of the sensing performance, $\mathrm{T}$ sensing period delay for the secondary user in order to detect the vacancy of the signal could be bearable in cognitive radio driven hospitals. Also, by choosing $\mathrm{T}$ and periodic sensing distance small enough, we can make the delay minimum. This research findings help to understand the effect of the memory in a sensing method and advantage and disadvantages of that which helps find optimal solutions to fulfil fundamental sensing requirements in IEEE 802.22 WRAN.

\section{References}

[1] I. A. Mamoon, A. K. M Muzahidul-Islam, Sabariah Baharun, Shozo Komaki, Ashir Ahmed, "Architecture and communication protocols for cognitive radio network enabled hospital" IEEE International Symposium on Medical Information and Communication Technology (ISMICT), 2015, pp. 170-174.

[2] I. A. Mamoon, A. K. M Muzahidul Islam, Ahmad Shahrizal Sani, Sabariah Baharun, Shozo Komaki Toshio Wakabayashi, "Definition, design and priority management of a cognitive radio driven hospital: CogMed", IEEE Conference on Biomedical Engineering and Sciences (IECBES), 2014, pp. 373-378.

[3] A. Eslami, S. Karamzadeh, "Performance Analysis of Energy Based Spectrum Sensing over Nakagami-m Fading Channels with Noise uncertainty", International Journal Of Electronics, Mechanical Aand Mechatronics Engineering (IJEMME), 2016, vol:6, pp: 1101-1106.

[4] A. Eslami, S. Karamzadeh, "Performance analysis of double threshold energy detection-based spectrum sensing in low SNRs over Nakagami-m fading channels with noise uncertainty", IEEE conference on Signal Processing and Communication Application (SIU), 2016, pp: 309-312.

[5] S. Atapattu, C. Tellambura And H. Jiang, "Spectrum sensing via energy detector in low snr", 2011 IEEE International Conference on Communications (ICC), June 2011, pp. 1-5.

[6] J. Zhu, Z. Xu, F. Wang, B. Huang, B. Zhang, "Double threshold energy detection of cooperative spectrum sensing in cognitive radio", IEEE CrownCom, pp. 15, Singapore, May 2008.

[7] N. Pillay and H.J. Xu, "Blind eigenvalue-based spectrum sensing for cognitive radio networks" IET Commun., 2012, Vol. 6, Iss. 11, pp. 1388-1396.

[8] N. Han, S. H. Shon, J. O. Joo, and J. M. Kim, "Spectral correlation based signal detection method for spectrum sensing in IEEE 802.22 WRAN systems," Intern. Conf. Advanced Commun. Technology, Korea, Feb. 2006.

[9] Y. Zeng and Y.-C. Liang, "Eigenvalue-based spectrum sensing algorithms for cognitive radio" , IEEE Trans. Commun., vol. 57 , no. 6 , pp.1784 -1793 , 2009.

[10] Z.Ye, G.Memik, and J.Grosspietsch, "Energy Detection using Estimated Noise Variance for Spectrum Sensing in Cognitive Radio Networks, " in Wireless Communications and Networking Conference 2008.Las Vegas, USA: IEEE Communications Society, Mar.-Apr.2008. 